\title{
Well-differentiated Hepatocellular Carcinoma Detected as Hypovascularity by Only CT during Hepatic Arteriography
}

\author{
Jun Saito ${ }^{1}$, Soo Ryang Kim ${ }^{1}$, Masatoshi Kudo ${ }^{2}$, Susumu Imoto ${ }^{1}$, Kenji Ando ${ }^{1}$, \\ Taisuke Nakajima ${ }^{1}$, Katsumi Fukuda ${ }^{1}$, Yumi Otono ${ }^{1}$, Soo Ki Kim ${ }^{3}$, Takamitsu Komaki ${ }^{4}$, \\ Hirohisa Yano ${ }^{5}$, Osamu Nakashima ${ }^{5}$, Kayo Sugimoto ${ }^{6}$ and Toshiyuki Matsuoka ${ }^{7}$
}

\begin{abstract}
We describe a well-differentiated hepatocellular carcinoma (HCC) with alcohol-related liver cirrhosis in a 69-year-old man. Ultrasonography (US) disclosed a $10 \mathrm{~mm}$ hypoechoic nodule in segment 4; Sonazoid contrast-enhanced US and gadolinium-ethoxybenzyl-diethylenetriamine pentaacetic acid (Gd-EOB-DTPA)enhanced magnetic resonance imaging (MRI) revealed no defect in either the Kupffer phase or the hepatobiliary phase. Computed tomography during hepatic arteriography (CTHA), however, revealed a hypovascular nodule, but CT during arterial portography showed no perfusion defect. Histological analysis indicated a well-differentiated HCC. Thus, our detection of well-differentiated HCC disclosed by only CTHA attested to the efficiency of this modality, suggesting that it is more sensitive than Gd-EOB-GTPA-enhanced MRI.
\end{abstract}

Key words: well-differentiated hepatocellular carcinoma, imaging studies, Gd-EOB-DTPA-enhanced MRI, CT during hepatic arteriography, hypovascular tumor, Sonazoid contrast-enhanced US

(Intern Med 51: 885-890, 2012)

(DOI: 10.2169/internalmedicine.51.6904)

\section{Introduction}

The definitive diagnosis of nodular lesions detected by imaging techniques in the cirrhotic liver remains a critical challenge for clinicians. The issue is particularly complicated for small $(1-2 \mathrm{~cm})$ nodules, many of which may be preneoplastic with uncertain malignant potential (1), such as macroregenerative nodules, low-grade dysplastic nodules (LGDN), high-grade dysplastic nodules (HGDN), or more rarely, hemangiomas that are found in up to $42 \%$ of explanted livers (2-4).

Recently, clinicians have been able to conduct computed tomography (CT) scanning during angiography, CT during hepatic arteriography (CTHA) and CT during arterial portography (CTAP), thereby simultaneously acquiring data on lesions and intranodular blood flow $(5,6)$. Moreover, devel- opment of the newly introduced diagnostic imaging techniques, Sonazoid contrast-enhanced ultrasonography (US) (7) and gadolinium-ethoxybenzyl-diethylenetriamine pentaacetic acid (Gd-EOB-DTPA)-enhanced magnetic resonance imaging (MRI) (8), have provided higher degrees of detectability of small hepatocellular carcinoma (HCC).

Here, we describe a $10 \mathrm{~mm}$ well-differentiated HCC appearing as a hypovascular tumor, with alcohol-related liver cirrhosis, disclosed by only CTHA, whereas Sonazoid contrast-enhanced US and Gd-EOB-DTPA-enhanced MRI revealed no defect in either the Kupffer phase or the hepatobiliary phase; also, contrast-enhanced CT revealed no washout in the equilibrium phase.

\section{Case Report}

A 69-year-old man with alcohol-related liver cirrhosis was

\footnotetext{
${ }^{1}$ Department of Gastroenterology, Kobe Asahi Hospital, Japan, ${ }^{2}$ Department of Gastroenterology and Hepatology, Kinki University School of Medicine, Japan, ${ }^{3}$ Department of Gastroenterology and Hepatology, Kyoto University, Japan, ${ }^{4}$ Department of Internal Medicine, SaiseikaiTondabayashi Hospital, Japan, ${ }^{5}$ Department of Pathology, Kurume University School of Medicine, Japan, ${ }^{6}$ Department of Pharmacy, Kobe Asahi Hospital, Japan and ${ }^{7}$ Department of Radiology, Osaka City University Medical School, Japan

Received for publication November 9, 2011; Accepted for publication January 5, 2012

Correspondence to Dr. Soo Ryang Kim, asahi-hp@arion.ocn.ne.jp
} 

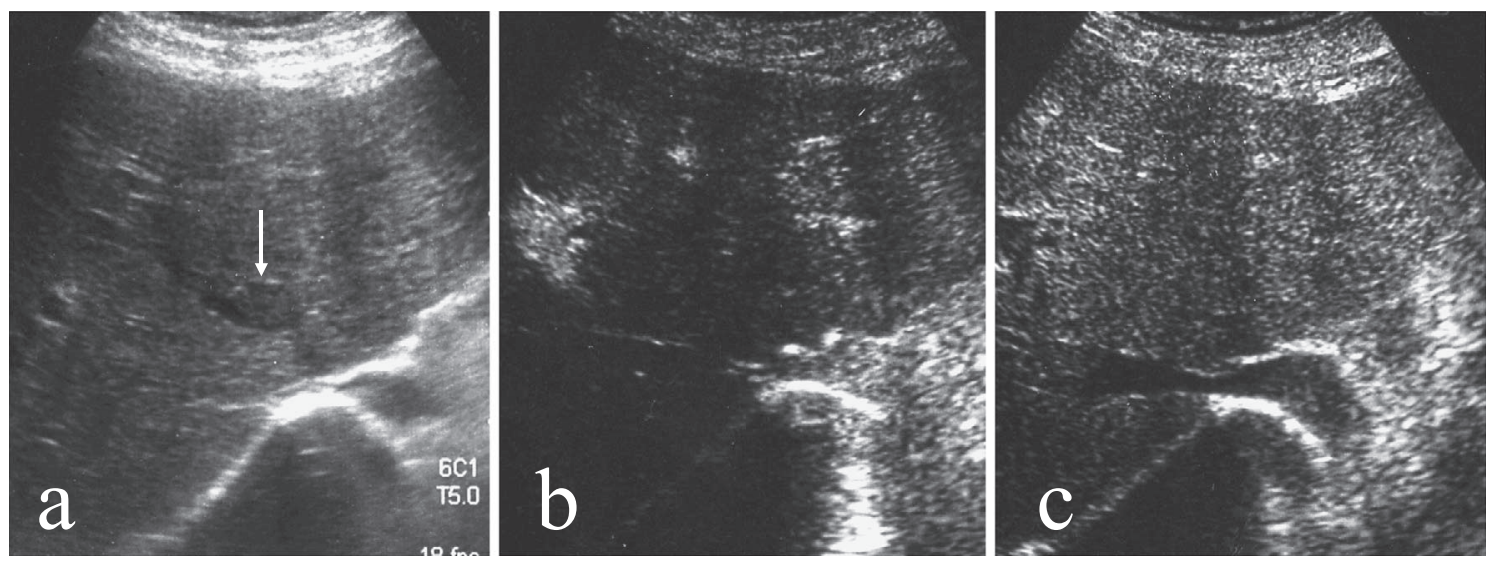

Figure 1. Imaging findings of US. (a) B-mode US reveals a $10 \mathrm{~mm}$ hypoechoic nodule in segment 4 (arrow). The hypovascular nodule is not visible in either (b) the early vascular phase (5 sec) or (c) the Kupffer phase (15 min) on Sonazoid contrast-enhanced US. Contrast-enhanced US imaging was performed with Xario SSA-660A, PVT-375BT probe (3.5 MHz) (Toshiba Medical Systems). PS-low; mechanical index: 0.2 , frame rate: $15 \mathrm{fps}$, dynamic range: $45 \mathrm{~dB}$, injection of Sonazoid: $0.0075 \mathrm{~mL} /$ kg

admitted to Kobe Asahi Hospital in July 2010 for further examination of a $10 \mathrm{~mm}$ hypoechoic nodule in segment four (S4). His alcohol consumption over 40 years was $360 \mathrm{~mL} /$ day. A physical examination on admission showed no remarkable abnormalities and no evidence of lymph adenopathy or splenomegaly. The serum was negative for hepatitis $\mathrm{C}$ virus antibody and hepatitis B surface antigen. Laboratory data disclosed the following values: platelets $10.2 \times 10^{4} / \mu \mathrm{L}$ (normal, 13.1-36.2), total protein $7.0 \mathrm{~g} / \mathrm{dL}$ (6.7-8.3), $\gamma-$ glutamyl transpeptidase $281 \mathrm{IU} / \mathrm{L}(0-30)$, albumin $4.2 \mathrm{~g} / \mathrm{dL}$ (4.0-5.0), total bilirubin $0.9 \mathrm{mg} / \mathrm{dL}$ (0.3-1.2), prothrombin time $82 \%$ without encephalopathy and ascites, classified as Child-Pugh classification A of cirrhosis. Alpha-fetoprotein (AFP), lens culinaris agglutinin A-reactive fraction of alpha fetoprotein (AFP L3) and protein-induced vitamin $\mathrm{K}$ absence (PIVKA II) were within normal ranges. A US-guided biopsy disclosed alcohol-related liver cirrhosis with pericellular fibrosis. B-mode US disclosed a $10 \mathrm{~mm}$ hypoechoic nodule in S4 (Fig. 1a). Sonazoid contrast-enhanced US revealed no hypervascular nodule in the early vascular phase (Fig. 1b) and no defect in the Kupffer phase (Fig. 1c). Plain CT showed no fatty liver or any nodule in the liver (Fig. 2a); contrast-enhanced CT revealed no enhanced nodule in the arterial phase (Fig. 2b) and no washout in the equilibrium phase (Fig. 2c); Gd-EOB-DTPA-enhanced MRI revealed no enhanced nodule in the arterial phase (Fig. 2d), and no defect in the hepatobiliary phase (Fig. 2e). CTHA, however, revealed a hypovascular nodule (Fig. 3a, b), but CTAP revealed no perfusion defect (Fig. 3c). A US-guided biopsy of the nodule was diagnosed as well-differentiated HCC characterized by more than two-fold the cellularity of the non-tumorous area, clear cell change and mild cell atypia (Fig. 4a, b). Immunohistochemical staining of CD34 in the nodule was positive in the sinusoidal blood space, implying capillarization compatible with well-differentiated
HCC (Fig. 4c, d). The organic anion transporter (OATP) 1B3 (9), being positive in both the nodule and the nonnodular lesion, was compatible with the absence of defect in the hepatobiliary phase as determined by Gd-EOB-DTPAenhanced MRI (Fig. 5a, b).

\section{Discussion}

Confirmation of arterial hypervascularity by three imaging modalities (triphasic CT, triphasic MRI, and contrastenhanced US), even in the absence of a significant ( $>400$ $\mathrm{ng} / \mathrm{mL}$ ) rise in $\alpha$-fetoprotein, is recommended by the European Association for the Study of the Liver as diagnostic criteria for $\mathrm{HCC}$ nodules larger than $2 \mathrm{~cm}$ in patients with cirrhosis (10). This recommendation for the management of HCC provides a rational approach to the problem but leaves some areas of uncertainty, particularly those concerning the interpretation of discordant vascularity, the use of imaging techniques in nodules smaller than $2 \mathrm{~cm}$, the meaning of truly hypovascular nodules, and the management of those diagnosed as LGDN or HGDN at guided biopsy.

To resolve the areas of uncertainty, we previously evaluated the superiority of CT arterioportal angiography for nodules smaller than $2 \mathrm{~cm}$, and concluded that the modality is superior to contrast-enhanced $\mathrm{CT}$ and contrast-enhanced MRI (11). Nonetheless, the status of imaging studies in the diagnosis of HCC smaller than $2 \mathrm{~cm}$ has changed with the introduction of new contrast agents for US and MRI. First, Sonazoid was exclusively approved in Japan in 2007 as a second-generation US contrast agent; second, Gd-EOBDTPA, a new liver-specific contrast agent used in MRI, was approved in 2008. Taking these improvements into consideration, we evaluated the effectiveness of four imaging modalities (contrast-enhanced CT, Sonazoid contrast-enhanced US, Gd-EOB-DTPA-enhanced MRI, CT arterioportal angi- 

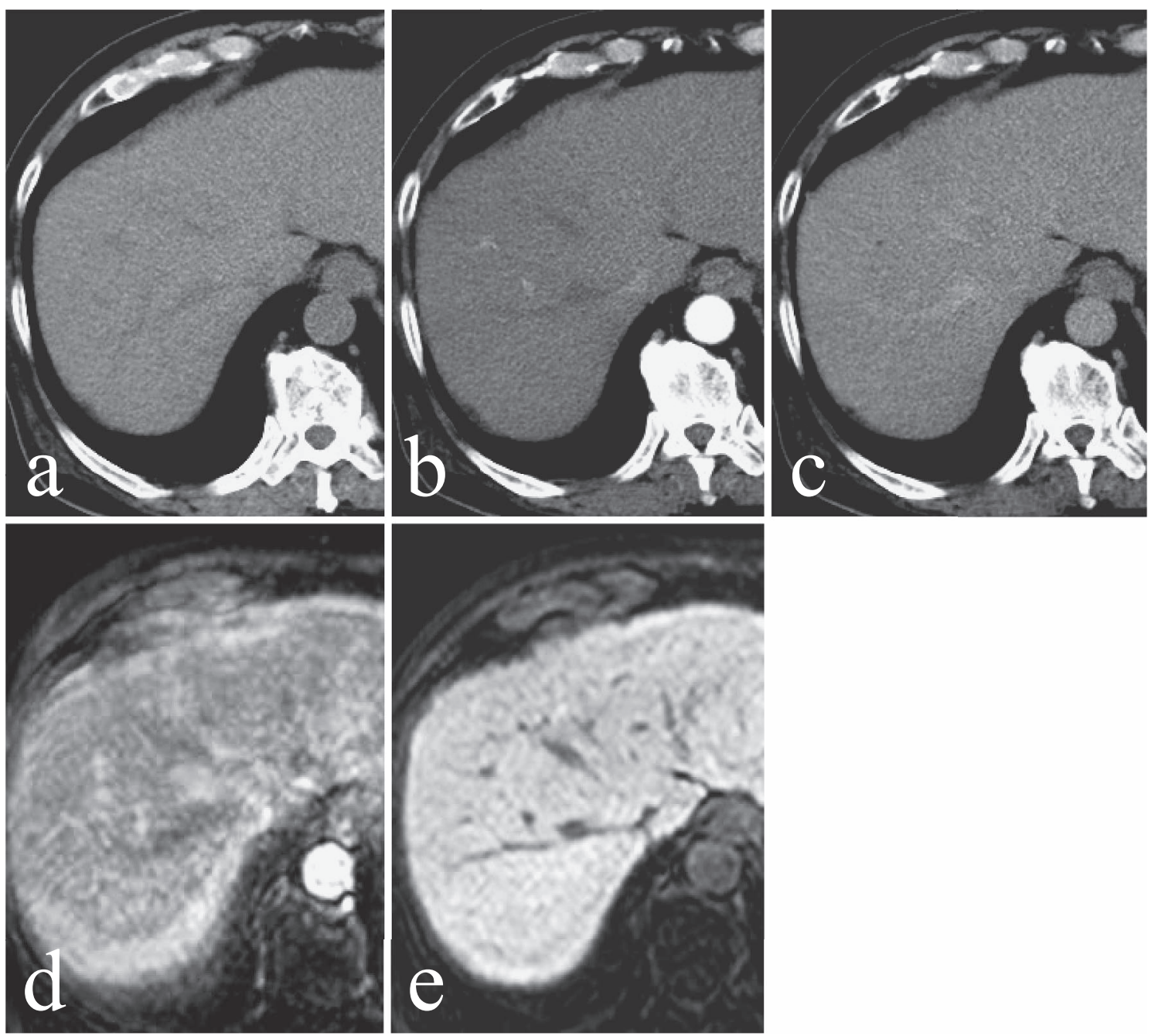

Figure 2. Imaging findings of CT and MRI. (a) Plain CT reveals no fatty liver or any nodule in the liver. (b) Contrast-enhanced CT reveals no enhanced nodule in the arterial phase and (c) no washout in the equilibrium phase. CT imaging was performed with Somatom Emotion 16-Slice configuration (Siemens Japan). (d) Gd-EOB-DTPA-enhanced MRI reveals no enhanced nodule in the arterial phase and (e) no defect in the hepatobiliary phase. MR imaging at the hepatobiliary phase was performed with Gyroscan 10T-NT (Philips Medical Systems), Intera 1.0T version 12.1. THRIVE; TR/TE: 5.4/2.8 msec, FA: $20^{\circ}$, SENSE factor: 2 , slice thickness: $6 \mathrm{~mm}$ (gap $\mathbf{- 2 . 5} \mathbf{m m}$ ), slice number: 70, matrix: $160 \times 512$

ography) in the diagnosis of HCC smaller than $2 \mathrm{~cm}$. Consequently, the following patterns disclosed by these imaging modalities were deemed to be a conclusive diagnosis of HCC: 1) hypervascularity in the arterial phase and washout in the equilibrium phase (by contrast-enhanced CT), 2) hypervascularity in the early vascular phase and defect in the Kupffer phase (by Sonazoid contrast-enhanced US), 3) hypervascularity in the arterial phase and/or defect in the hepatobiliary phase (by Gd-EOB-DTPA-enhanced MRI) and 4) hypervascularity by CTHA and/or perfusion defect by CTAP (by CT arterioportal angiography). The results showed that the diagnostic sensitivity of CT arterioportal angiography was $88.2 \%$ in all nodules and $95.8 \%$ in moderatelydifferentiated HCC, with a significant difference between contrast-enhanced CT and CT arterioportal angiography $(\mathrm{p}<$ 0.05 ). No difference was observed, however, among Sona- zoid contrast-enhanced US, Gd-EOB-DTPA-enhanced MRI and CT arterioportal angiography. The combined sensitivity of Sonazoid contrast-enhanced US and Gd-EOB-DTPAenhanced MRI in all nodules was $94.1 \%$ due to improvement in the diagnostic capabilities of each modality (12). For the diagnosis of nodules smaller than $2 \mathrm{~cm}$, these two imaging modalities have provided higher sensitivity with Sonazoid CEUS and Gd-EOB-DTPA-enhanced MRI than with Sonovue contrast-enhanced US and contrast-enhanced CT (13), or with Sonovue contrast-enhanced US and gadolinium-enhanced MRI (14). Such sensitivity in the diagnosis of well-differentiated HCC was, however, not explicit with the use of those imaging modalities in the present study.

According to the classification by the International Working Party of the World Congress of Gastroenterology, he- 

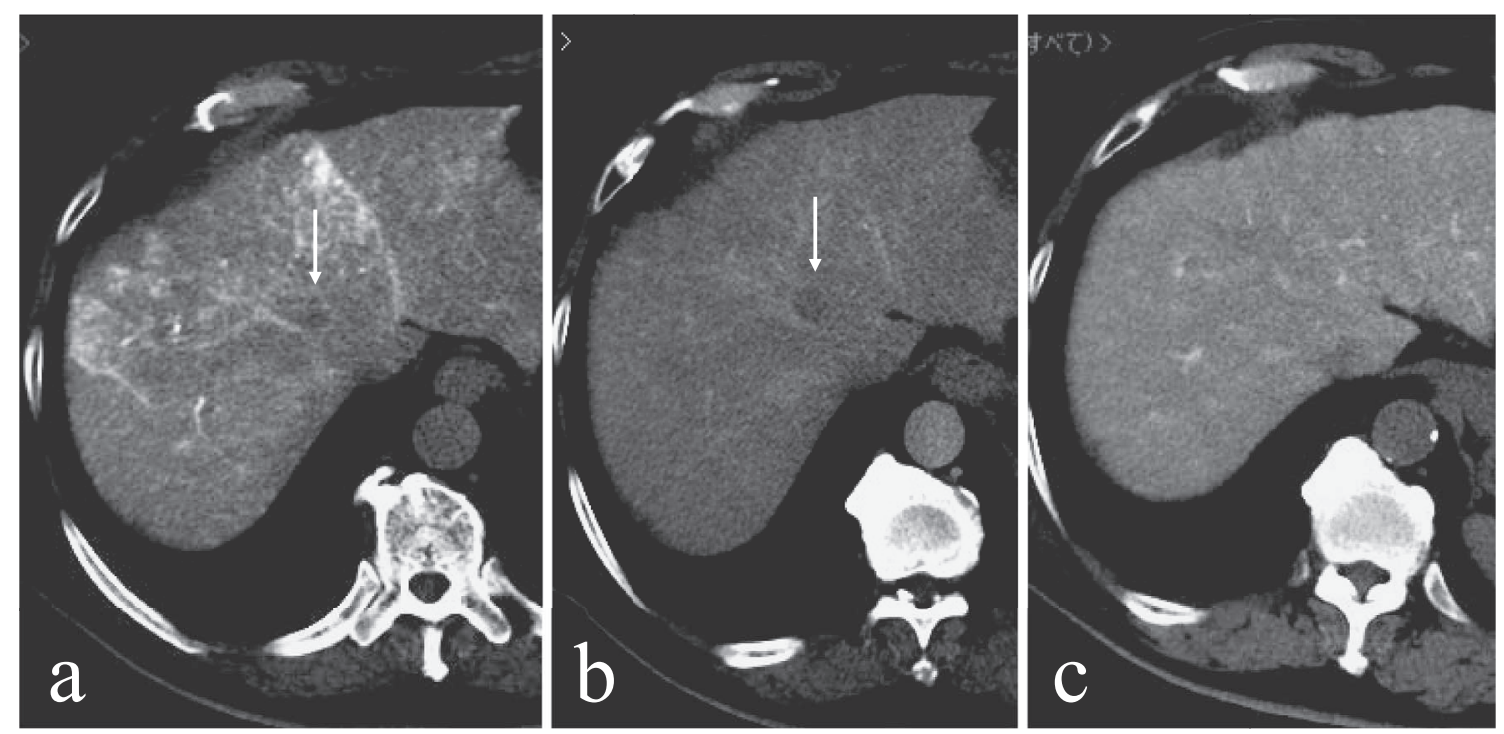

Figure 3. Imaging findings of CT arterioportal angiography. CTHA reveals a hypovascular nodule in both (a) the early phase (arrow) and (b) the late phase (arrow), (c) although CTAP does not reveal any perfusion defect. CTHA was performed using $40-60 \mathrm{~mL}$ of contrast medium at the rate of 2-3 $\mathrm{mL} / \mathrm{s}$ into the common hepatic artery, scanned early phase (15 s) and late phase (60 s). CTAP was performed using $80-90 \mathrm{~mL}$ of contrast medium at the rate of $2-3 \mathrm{~mL} / \mathrm{s}$.

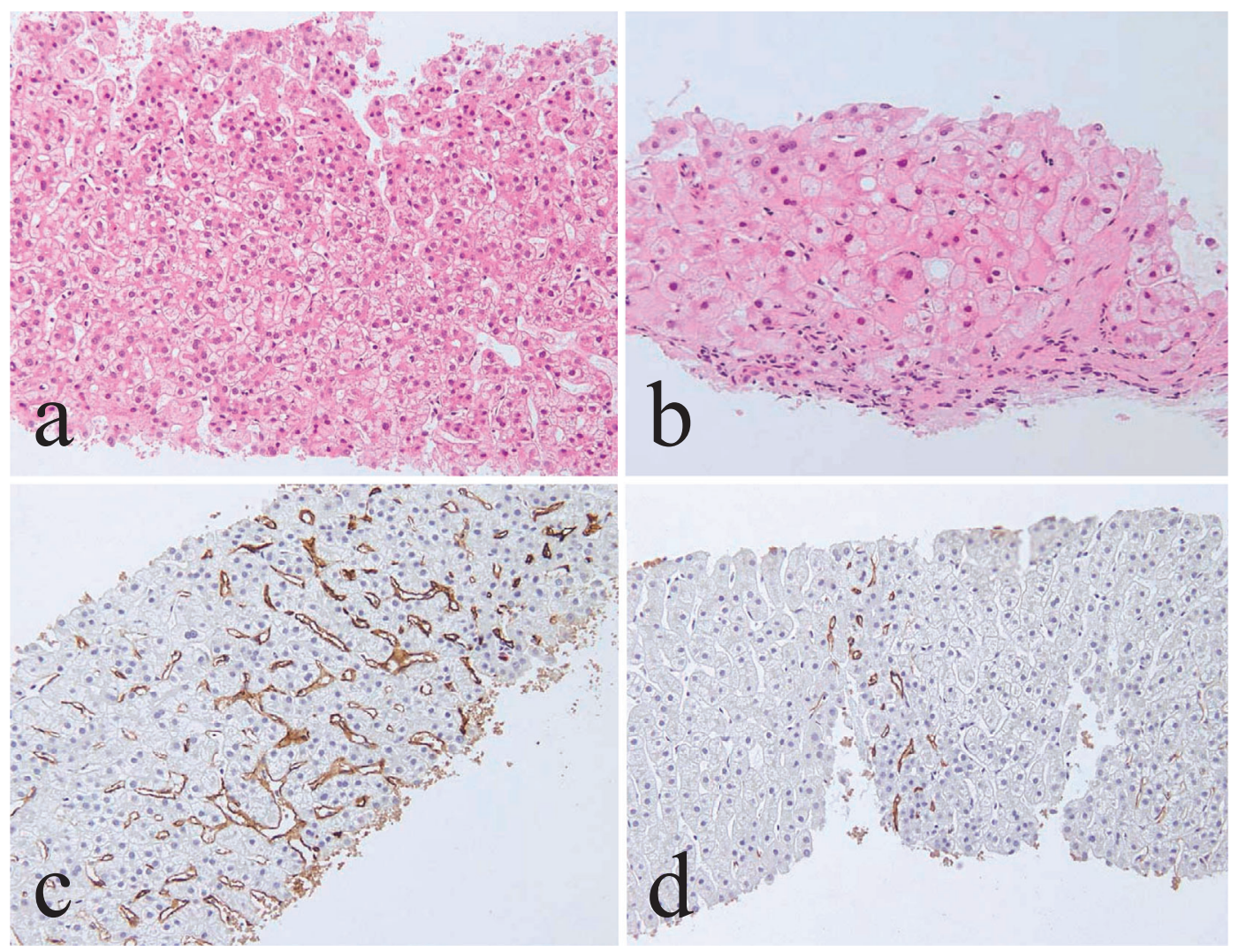

Figure 4. Histological findings of the US-guided biopsy specimens. (a) Well-differentiated HCC characterized by more than two-fold the cellularity of the non-tumorous area, clear cell change and mild cell atypia, and (b) non-nodular lesion (Hematoxylin and Eosin staining). (c) Capillarization is evident in the sinusoidal blood space, and (d) non-nodular lesion (immunohistochemical staining by CD34).

patic nodules in patients with chronic liver diseases are subdivided into regenerative nodules (mono acinus and multi acinus ), LGDN, HGDN, well-differentiated HCC, moderately-differentiated $\mathrm{HCC}$ and poorly-differentiated 


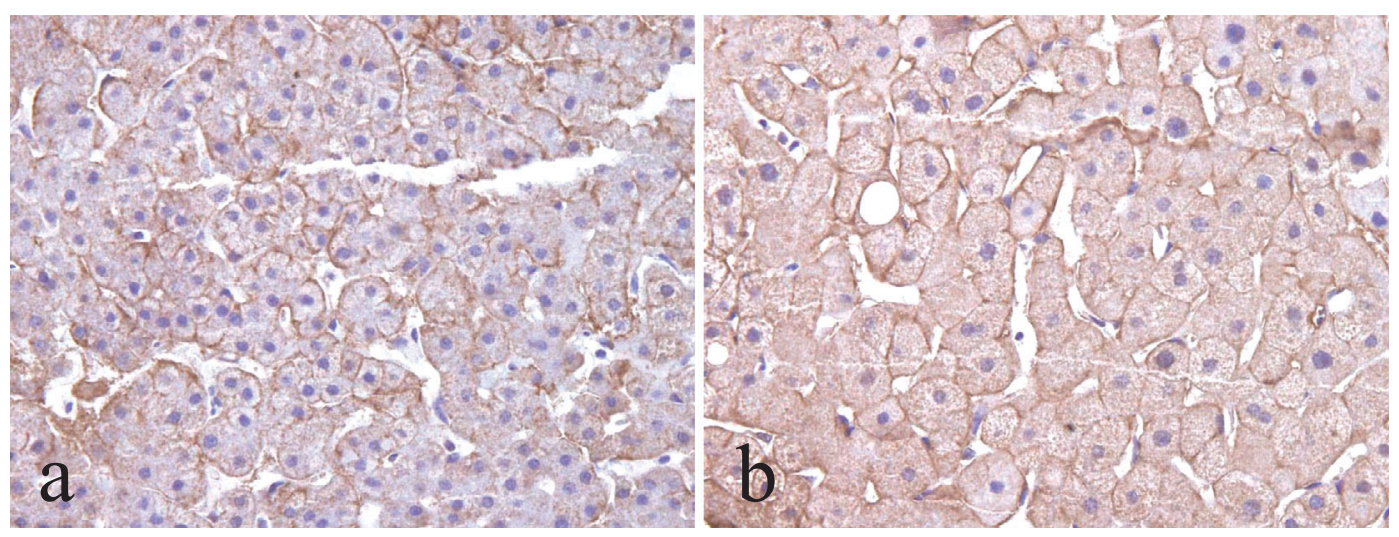

Figure 5. Expression of OATP 1 B3 is observed in both (a) the nodular lesion and (b) the non-nodular lesion.

$\mathrm{HCC}$, in ascending order of histologic grades, representing a sequence of multistep hepatocarcinogenesis (1). In a sequence of multistep carcinogenesis, the differentiation of HGDN from well-differentiated HCC is pivotal. From the viewpoint of histopathology, consensus on the criteria of well-differentiated HCC has been established (15). From the viewpoint of imaging studies on early HCC, there is controversy among hepatologists engaged in the diagnosis of welldifferentiated HCC. Some insist that Gd-EOB-DTPAenhanced MRI is most sensitive in disclosing defect in the hepatobiliary phase (16) and others insist that CTAP with CT arterioportal angiography is the most sensitive in disclosing perfusion defect (11). Although defect disclosed by CTHA, implying hypovascular HCC, has been reported to occur before perfusion defect disclosed by CTAP (6), the finding has not been verified in the clinical setting. We described a $10 \mathrm{~mm}$ well-differentiated HCC disclosed by only CTHA as a hypovascular tumor, whereas Sonazoid contrastenhanced US, contrast-enhanced CT and Gd-EOB-DTPAenhanced MRI revealed no defect in the Kupffer phase and no washout in the equilibrium phase and no defect in the hepatobiliary phase; also, CTAP revealed no perfusion defect.

Concerning OATP 1B3, there was no difference of OATP 1B3 expression in either the nodule or the non-nodular lesion in the present case of well-differentiated HCC. That is compatible with two reports, describing that OATP 1B3 was highly expressed in some of moderately-differentiated HCC, whereas it was hardly observed in well-differentiated HCC $(9,17)$. Concerning insufficient quality of the images, the unhomogeneous enhanced area depicted with CTHA and Sonazoid contrast-enhanced US might be explained by pericellular fibrosis due to alcohol-related liver cirrhosis.

The present results demonstrated that CTHA is more sensitive than Gd-EOB-GTPA-enhanced MRI in the diagnosis of well-differentiated HCC, although no clinical trials have been undertaken to evaluate the sensitivity of the two above modalities in the diagnosis of well-differentiated HCC. Further study is needed to clarify the sensitivity of imaging studies in the diagnosis of well-differentiated HCC smaller than $2 \mathrm{~cm}$.

The authors state that they have no Conflict of Interest (COI).

\section{Acknowledgement}

We are indebted to Ms. Yoshiko Kawamura of Kobe Asahi Hospital for the preparation of the manuscript.

\section{References}

1. International Working Party. Terminology of nodular hepatocellular lesions. Hepatology 22: 983-989, 1995.

2. Theise N, Schwartz M, Miller C, Thung S. Macroregenerative nodules and hepatocellular carcinoma in forty-four sequential adult liver explants with cirrhosis. Hepatology 16: 949-955, 1992.

3. Mion F, Grozel L, Boillot O, Paliard P, Berger F. Adult cirrhotic liver explants: precancerous lesions and undetected small hepatocellular carcinomas. Gastroenterology 111: 1587-1592, 1996.

4. Burrel M, Llovet J, Ayuso C, et al. MRI angiography is superior to helical CT for detection of HCC prior to liver transplantation: an explant correlation. Hepatology 38: 1034-1042, 2003.

5. Hayashi M, Matsui O, Ueda K, Kawamori Y, Gabata T, Kadoya M. Progression to hypervascular hepatocellular carcinoma: correlation with intranodular blood supply evaluated with CT during intraarterial injection of contrast material. Radiology 225: 143-149, 2002.

6. Tajima $\mathrm{T}$, Honda $\mathrm{H}$, Taguchi $\mathrm{K}$, et al. Sequential hemodynamic change in hepatocellular carcinoma and dysplastic nodules: CT angiography and pathologic correlation. AJR Am J Roentgenol 178: 885-897, 2002.

7. Inoue $\mathrm{T}$, Kudo $\mathrm{M}$, Hatanaka $\mathrm{K}$, et al. Imaging of hepatocellular carcinoma: qualitative and quantitative analysis of postvascular phase contrast enhanced ultrasonography with sonazoid. Comparison with superparamagnetic iron oxide magnetic resonance images. Oncology 75: 48S-54S, 2008.

8. Saito K, Kotake F, Ito N, et al. Gd-EOB-DTPA enhanced MRI for hepatocellular carcinoma: quantitative evaluation of tumor enhancement in hepatobiliary phase. Magn Reson Med Sci 4: 1-9, 2005.

9. Narita M, Hatano E, Arizono S, et al. Expression of OATP1B3 determines uptake of Gd-EOB-DTPA in hepatocellular carcinoma. J Gastroenterol 44: 793-798, 2009.

10. Bruix J, Sherman M, Llovet J, et al. Clinical management of hepatocellular carcinoma. Conclusions of the Barcelona-2000 EASL conference. J Hepatol 35: 421-430, 2001.

11. Kim SR, Ando K, Mita K, et al. Superiority of CT arterioportal 
angiography to contrast-enhanced CT and MRI in the diagnosis of hepatocellular carcinoma in nodules smaller than $2 \mathrm{~cm}$. Oncology 72: 58S-66S, 2007.

12. Mita K, Kim SR, Kudo M, et al. Diagnostic sensitivity of imaging modalities for hepatocellular carcinoma smaller than $2 \mathrm{~cm}$. World J Gastroenterol 16: 4187-4192, 2010.

13. Bolondi L, Gaiani S, Celli N, et al. Characterization of small nodules in cirrhosis by assessment of vascularity: the problem of hypovascular hepatocellular carcinoma. Hepatology 42: 27-34, 2005.

14. Forner A, Vilana R, Ayuso C, et al. Diagnosis of hepatic nodules $20 \mathrm{~mm}$ or smaller in cirrhosis: prospective validation of the noninvasive diagnostic criteria for hepatocellular carcinoma. Hepatology 47: 97-104, 2008.
15. International Consensus Group for Hepatocellular Neoplasia. Pathologic diagnosis of early hepatocellular carcinoma: a report of the International Consensus Group for Hepatocellular Neoplasia. Hepatology 49: 658-664, 2009.

16. Motosugi U, Ichikawa $\mathrm{T}$, Sou $\mathrm{H}$, et al. Liver parenchymal enhancement of hepatocyte-phase images in Gd-EOB-DTPAenhanced MR imaging: which biological markers of the liver function affect the enhancement? J Magn Reson Imaging 30: 1042-1046, 2009.

17. Kitao A, Matsui O, Yoneda $\mathrm{N}$, et al. The uptake transporter OATP8 expression decreases during multistep hepatocarcinogenesis: correlation with gadoxetic acid enhanced MR imaging. Eur Radiol 21: 2056-2066, 2011.

(C) 2012 The Japanese Society of Internal Medicine http://www.naika.or.jp/imindex.html 